PROCEEDINGS OF THE

AMERICAN MATHEMATICAL SOCIETY

Volume 129, Number 1, Pages 211-217

S 0002-9939(00)05512-X

Article electronically published on July 27, 2000

\title{
POLYNOMIAL SOLUTIONS TO DIRICHLET PROBLEMS
}

\author{
MARC CHAMBERLAND AND DAVID SIEGEL
}

(Communicated by Albert Baernstein II)

Abstract. The Dirichlet problem

$$
\begin{aligned}
\Delta u(x, y) & =0 \text { in } \mathbb{R}^{2}, \\
u(x, y) & =f(x, y) \text { on } \psi(x, y)=0
\end{aligned}
$$

is considered where the functions $f$ and $\psi$ are polynomials. The authors study the problem of determining which functions $\psi$ will admit polynomial solutions $u$ for any polynomial $f$. When one additionally requires the classical condition $\operatorname{deg} u \leq \operatorname{deg} f$, this forces $\operatorname{deg} \psi \leq 2$, and a complete classification is obtained. Some necessary conditions are obtained for the case $\operatorname{deg} \psi>2$.

\section{INTRODUCTION}

In this paper, we consider the system

$$
\begin{aligned}
\Delta u(x, y) & =0 \text { in } \mathbb{R}^{2}, \\
u(x, y) & =f(x, y) \text { on } \psi(x, y)=0,
\end{aligned}
$$

where $f(x, y)$ and $\psi(x, y)$ are polynomials, and $\Delta=\partial^{2} / \partial x^{2}+\partial^{2} / \partial y^{2}$ is the Laplacian operator. Throughout the paper, we will let $\mathcal{P}$ denote the set of all polynomials, $\mathcal{P}_{m}$ the set of polynomials of degree at most $m$, and $\mathcal{P}_{m}^{\prime}$ the set of homogeneous polynomials of degree $m$.

This paper studies the following

Basic Problem. For a given polynomial $\psi$, is there a polynomial solution $u \in \mathcal{P}$ to the system (11)-(2) for any polynomial "data" $f \in \mathcal{P}$ ?

We contrast this to the

Restricted Problem. For a given polynomial $\psi$, is there a polynomial solution $u \in \mathcal{P}_{m}$ to the system (1)-(2) for any polynomial "data" $f \in \mathcal{P}_{m}$ ?

The restricted problem has been studied extensively (see for example [4], [6] and [7]). This type of problem was studied in $\mathbb{R}^{n}$ with entire data (hence requesting entire solutions) by Shapiro [8] and by Khavinson and Shapiro [9]. Axler [1] documents the classical work done when $\psi=0$ represents an $n$-sphere and we restrict ourselves again to polynomials, and gives fast algorithms [2] for solving such problems.

This work focuses on the Basic Problem in $\mathbb{R}^{2}$. The Basic Problem differs significantly from the Restricted Problem; they will be compared throughout the paper.

Received by the editors February 20, 1998 and, in revised form, April 5, 1999.

2000 Mathematics Subject Classification. Primary 31A25.

(C)2000 American Mathematical Society 
Section 2 shows that we need only consider the so-called "square-free" functions $\psi$. We then extend an argument of Khavinson and Shapiro [9], indicating that only square-free $\psi$ with $\operatorname{deg} \psi \leq 2$ need be considered for the Restricted Problem. This paves a natural path for Section 3 which exhaustively considers the Basic Problem when $\operatorname{deg} \psi \leq 2$. Lastly, Section 4 considers the case when $\operatorname{deg} \psi>2$ and gives some necessary conditions which an affirmative answer to the Basic Problem would require.

\section{SquARE-FreE POLYNOMIALS}

Since $\psi(x, y)$ is a polynomial, the equation $\psi(x, y)=0$ will describe a set of algebraic curves in the plane on which we require $u(x, y)=f(x, y)$. Factoring $\psi$ into irreducible components, it is clearly redundant to have two irreducible terms which are proportional, so we shall ignore such cases. We can then write $\psi=\psi_{1} \cdots \psi_{m}$. We assume the non-degeneracy condition that each curve $\psi_{i}(x, y)=0$ has infinitely many points. We shall denote these possible $\psi$ 's as square-free polynomials. Of course the irreducible polynomials are a subset of the square-free polynomials.

Assuming $\psi$ is irreducible (and non-degenerate), Khavinson and Shapiro [9] state that if for every $f \in \mathcal{P}_{m}$ there is a solution $u \in \mathcal{P}_{m}$ for the problem (1)-(2), then $u-f=\psi g$ where $g$ is a polynomial. We generalize this to the following:

Theorem 2.1. Suppose that $\psi$ is square-free. Then if for $f \in \mathcal{P}$ there is a solution $u \in \mathcal{P}$ for the problem (11) -(21), then $u-f=\psi g$ where $g \in \mathcal{P}$.

Proof. Write $\psi=\psi_{1} \cdots \psi_{m}$, where each curve $\psi_{i}(x, y)=0$ has infinitely many points. Then by the Lemma on page 2 of Shafarevich [11], $u-f$ is divisible by $\psi_{i}$ for every $i$. It then follows that $u-f$ is divisible by $\psi$.

In considering the Restricted Problem, Khavinson and Shapiro [9] showed that the only irreducible $\psi$ admitting solutions in this class must have $\operatorname{deg} \psi \leq 2$. This implies that ellipsoids are the only bounded domains which yield solutions, in this limited sense (Ebenfelt 3] proved this in dimension 2). We repeat their elegant argument here, with an extension to square-free $\psi$.

Theorem 2.2. Suppose that $\psi$ is square free and $m \geq 2$. Then if for all $f \in \mathcal{P}_{m}$ there is a solution $u \in \mathcal{P}_{m}$ for the problem (11)-(2), then $\operatorname{deg} \psi \leq 2$.

Proof. Using Theorem 2.1. we have that if $u, f \in \mathcal{P}_{m}$ and $\psi \in \mathcal{P}_{k}$, then $u-f=\psi g$ where $g \in \mathcal{P}_{m-k}$ and $k \leq m$. Since $\left\{\Delta f: f \in \mathcal{P}_{m}\right\}=\mathcal{P}_{m-2}$, this implies the map $L: g \rightarrow \Delta(\psi g)$ is surjective from $\mathcal{P}_{m-k}$ to $\mathcal{P}_{m-2}$, thus yielding that $k \leq 2$.

\section{Quadratic CASES}

This section yields a complete analysis of the Basic Problem when $\operatorname{deg} \psi \leq 2$. In summary, we prove the following:

Theorem 3.1. When $\operatorname{deg} \psi \leq 2$, the set $\psi=0$ describes one of the following geometric cases, along with its status with regards to the Basic Problem:

(1) One Line: There is always a non-unique solution.

(2) Parallel Lines: There is always a unique solution.

(3) Parabolas: There is always a unique solution.

(4) Ellipses: There is always a unique solution. 
(5) Crossing Lines: There exists a unique solution if the angle between the crossing lines is incommensurate with $\pi$; otherwise there exist data with no corresponding solution.

(6) Hyperbolas: There exists a unique solution if the angle between the asymptotes is incommensurate with $\pi$; otherwise there exist data with no corresponding solution.

Before going case by case through the proof, some comments are in order. First, by considering the level curves of harmonic functions, Flatto et al. [5] proved the uniqueness results for cases (2)-(6). When the appropriate angle in cases (5) and (6) is commensurate with $\pi$, they showed non-uniqueness. Theorem 3.1 shows that for appropriately chosen data of the form $f=x^{n}$ there exists no corresponding polynomial solution. Flatto et al. also raised the existence question for entire data and treated it explicitly for case (3). Second, if Theorem 3.1 was considered for the Restricted Problem, existence results would follow from the uniqueness results immediately: $\mathcal{P}_{m}$ is a finite-dimensional space, so surjectivity of the map $L: g \rightarrow \Delta(\psi g)$ is equivalent to injectivity. This argument yields cases (2)-(4) and cases (5) and (6) when the appropriate angle is incommensurate with $\pi$. Third, the non-existence result in case (5) can be obtained by using that $L$ maps $\mathcal{P}_{m}^{\prime}$ to $\mathcal{P}_{m}^{\prime}$. This does not apply in case (6), so the non-existence result must be proven another way. The non-existence result in case (6) is the only part of Theorem 3.1 that cannot be obtained by combining the results of Flatto et al. with consideration of the operator $L$. Last, the following proof implicitly gives a recursive method for constructing solutions when existence holds.

Proof. Since the set of harmonic polynomials as well as $\mathcal{P}_{m}$ are both translation and rotation invariant, we will choose appropriate representations of each class enumerated in the statement of the theorem. Except in the case of one line, we will write $\psi=0$ as $y^{2}=h(x)$ for some polynomial $h(x)$, so all data $f$ (which is evaluated along the $\psi=0$ ) may be reduced to the form $f(x, y)=f_{1}(x)+f_{2}(x) y$. In this case $\psi$ is even in $y$ so that if $(x, y)$ is on the curve, then so is $(x,-y)$. Writing a harmonic solution $u$ of (1)-(2) as

$$
u(x, y)=A_{0}+\sum_{k=1}^{N}\left[A_{k} \Re(x+i y)^{k}+B_{k} \Im(x+i y)^{k}\right],
$$

and evaluating this at $(x, \pm y)$ gives

$$
f_{1}(x) \pm f_{2}(x) y=A_{0}+\sum_{k=1}^{N}\left[A_{k} \Re(x+i y)^{k} \pm B_{k} \Im(x+i y)^{k}\right]
$$

implying

$$
f_{1}(x)=A_{0}+\sum_{k=1}^{N} A_{k} \Re(x+i y)^{k}, f_{2}(x)=\sum_{k=1}^{N} B_{k} \frac{\Im(x+i y)^{k}}{y}
$$

for all $(x, y)$ on the curve $\psi(x, y)=0$. Note that $\Re(x+i y)^{k}$ is even in $y$ and $\Im(x+i y)^{k}$ is odd in $y$ for all $k$. Thus $\frac{\Im(x+i y)^{k}}{y}$ is a polynomial in $x$ and $y$ which is even in $y$ for all $k$. 
One Line. Without loss of generality, take the one line as $y=0$. One solution is $u(x, y)=\Re f(x+i y, 0)$. The solution is not unique since one may add $\Im(x+i y)^{m}$ to the solution, where $m$ is any positive integer.

Parallel Lines. Without loss of generality, consider the parallel lines $\psi=y^{2}-1$. If the highest order of $f$ contains terms of the form $x^{n}$, start by constructing the harmonic function (solution) with $\Re(x+i y)^{n}$ to match these highest order terms in the data along $\psi=0$. The extra terms will be of lower order. Similarly, if some of the highest order terms of $f$ are of the form $x^{n} y$, then construct the harmonic function by $\Im(x+i y)^{(n+1)} /(n+1)$ to match the highest order terms in the data along $\psi=0$. Again the extra terms will be of lower order. One may repeat these steps for the successive lower order terms so that a solution $u$ will be generated which matches the data.

Concerning uniqueness, it suffices to show that only the zero solution matches the data $f=0$. By (3i) evaluated at $y=1, A_{k}=0$ and $B_{k}=0$ for all $k$. Hence the solution $u$ must be identically zero.

Parabolas. Without loss of generality, consider the parabolas $\psi=y^{2}-x$. This case is virtually identical to that of the parallel lines. Considering highest order terms in the data of the form $x^{n}$, take the harmonic function $\Re(x+i y)^{n}$. If the data contains $x^{n} y$ in the highest order, consider $\Im(x+i y)^{(n+1)} /(n+1)$. As in the parallel lines case, we may successively construct a solution. The uniqueness part comes from replacing $y^{2}$ by $x$ in (31) and arguing as in the parallel lines case.

We note that the question of uniqueness could be addressed without the preceeding analysis by using the more sophisticated Phragmèn-Lindelöf Theory. The argument is as follows. We wish to show that a polynomial $u(x, y)$ which vanishes on the parabola $x=y^{2}$ must be identically zero. Consider the region $D=\left\{(x, y): x>y^{2}\right\}$. Let $N=\operatorname{deg} u$, and choose $\alpha>N$. We may suppose that $N \geq 1$. The region $D$ is contained in a sector $S$ with opening angle $\frac{\pi}{\alpha}$. By the Phragmèn-Lindelöff Principle (Theorem 18 on page 94 and Remark (ii) on page 96 of Protter and Weinberger [10]) we have that $u$ is identically zero in $D$. We must then have that $u$ is identically zero everywhere.

Ellipses. Without loss of generality, consider the ellipse $x^{2} / a^{2}+y^{2} / b^{2}=1$. Note that along the curve,

$$
\Re(x+i y)^{k}=x^{k} \sum_{j=0}^{\lfloor k / 2\rfloor}\left(\begin{array}{c}
k \\
2 j
\end{array}\right) b^{2 j} / a^{2 j}+\text { lower order terms. }
$$

This coefficient of $x^{k}$ is always positive, hence we may always construct the data $f_{1}(x)$ with a solution of the form $\sum_{k=0}^{N} A_{k} \Re(x+i y)^{k}$. Similarly, if the data is of the form $f_{2}(x) y$, we can always construct a solution of the form $\sum_{k=0}^{N} B_{k} \Im(x+i y)^{k}$.

Instead of proving uniqueness as we have done earlier, we simply cite the wellknown result that a Dirichlet problem has a unique solution on bounded domains.

Crossing Lines. This case is more delicate than the previous cases. Without loss of generality, consider the crossing lines $x^{2} / a^{2}-y^{2} / b^{2}=0$, or $y= \pm b x / a$, where $a, b>0$. 
If we take the data $f=x^{n}$, by (B)

$$
x^{n}=\sum_{k=0}^{N} A_{k} \Re(x+i y)^{k}
$$

on the curve. This gives $1=A_{n} \Re(1+b i / a)^{n}$. Letting $a+i b=r e^{i \theta}$, we cannot solve for $A_{n}$ if and only if $n \theta=m 2 \pi \pm \pi / 2$. Incidentally, the angle $\theta$ is equal to half the angle between the crossing lines. Similarly, with data $x^{n-1} y$, we cannot solve for $B_{n}$ if and only if $n \theta=m \pi$. Thus, a solution will exist if and only if the angle between the crossing lines is incommensurate with $\pi$. Uniqueness follows as in the case of parallel lines.

Hyperbolas. This is the most difficult case to prove. Without loss of generality, consider the hyperbola $x^{2} / a^{2}-y^{2} / b^{2}=4$, where $a, b>0$. In attempting to imitate the case of the ellipse, we fail because a factor of $(-1)^{j}$ enters into the formula (4), hence the coefficient to $x^{k}$ may be zero. We approach this case a different way.

We may also assume that $a^{2}+b^{2}=1$. Let us parametrize one branch of the curve as

This implies

$$
x=a\left(t+\frac{1}{t}\right), y=b\left(t-\frac{1}{t}\right) .
$$

$$
x+i y=w+\frac{1}{w},
$$

where $w=e^{i \theta} t, a+i b=e^{i \theta}$. This allows us to write

$$
(x+i y)^{k}=\sum_{j=0}^{\lfloor k / 2\rfloor}\left(\begin{array}{c}
k \\
j
\end{array}\right)\left[t^{2 j-k} e^{i \theta(2 j-k)}+t^{k-2 j} e^{i \theta(k-2 j)}\right]-d_{k}
$$

where

$$
d_{k}=\left\{\begin{array}{cc}
\left(\begin{array}{c}
k \\
k / 2
\end{array}\right), & k \text { even } \\
0, & k \text { odd }
\end{array}\right.
$$

This reduces to

$$
\Re(x+i y)^{k}=\sum_{j=0}^{\lfloor k / 2\rfloor}\left(\begin{array}{c}
k \\
j
\end{array}\right) \cos ((2 j-k) \theta)\left[t^{2 j-k}+t^{k-2 j}\right]-d_{k}
$$

and

$$
\Im(x+i y)^{k}=\sum_{j=0}^{\lfloor k / 2\rfloor}\left(\begin{array}{c}
k \\
j
\end{array}\right) \sin ((2 j-k) \theta)\left[t^{2 j-k}-t^{k-2 j}\right] .
$$

The angle $\theta$, which is half the angle between the asymptotes of the hyperbola, is commensurate with $\pi$ if and only if there are positive integers $R$ and $M$ such that $R \theta=\pi M$. This holds if and only if the term

$$
t^{R}-t^{-R}
$$

cannot be obtained. Along the branch of the hyperbola, this corresponds to the data

$$
(x / 2 a+y / 2 b)^{R}-(x / 2 a-y / 2 b)^{-R} .
$$

Uniqueness follows as in the parallel lines case. 


\section{NON-QUADRATIC CASES}

This section addresses the Basic Problem when $\operatorname{deg} \psi>2$. As seen from Section 2 , an affirmative answer to the Basic Problem may only occur if $\operatorname{deg} f<\operatorname{deg} u$ for at least one data $f$, so care must be taken when comparing the Basic Problem to the Restricted Problem. The main result of this section follows. This theorem and its proof are comparable to Theorem 2.1 of [5].

Theorem 4.1. Suppose $\operatorname{deg} \psi>2$ and $\psi$ is square-free. Then the Basic Problem may be answered affirmatively only if the highest order part of $\psi$, which we denote as $\psi_{N}$ of order $N$, factors into real linear terms, namely,

$$
\psi_{N}=\prod_{j=0}^{N}\left(a_{i} x-b_{i} y\right)
$$

for real numbers $a_{i}, b_{i}$, where the angles between the lines $a_{i} x-b_{i} y=0$, for all $i$, are positive integral multiples of $\frac{\pi}{M}$, for some integer $M, M>2$. Also, the lowest order term of $\psi$ has degree less than 3.

Proof. Theorem 2.1 states that for square-free $\psi$ we have $u-f=\psi g$ for some polynomial $g$. If $\operatorname{deg} f<\operatorname{deg} u$, then the highest order term of $\psi g$ must equal the highest order term of $u$, which is harmonic. Since a homogeneous harmonic polynomial always factors into linear parts, this forces the highest order term of $\psi$ to factor into linear parts which are a subset of the linear parts of the the factors of the highest order term of $u$. Let $M$ be the degree of $u$. Then the zero set of the highest order term of $u$ is a set of $M$ lines through the origin with angle $\frac{\pi}{M}$ between adjacent lines. Thus the first part of the theorem is proved.

To show that the lowest order term of $\psi$ is less than 3 , argue by contradiction. If this degree was 3 or larger, there is no way to generate the data $f=x^{2}$.

Corollary 4.1.1. The only bounded sets $\psi=0$ which yield an affirmative answer to the Basic Problem are ellipses.

Proof. If $\operatorname{deg} \psi>2$, then the highest order term of $\psi$ factors into real linear parts as in Theorem 4.1, hence the set $\psi=0$ is unbounded. This leaves only the case where $\operatorname{deg} \psi \leq 2$; the classification in the preceeding section completes the proof here.

Example 4.1. The curve defined by $\psi=\frac{x^{4}}{a^{2}}+\frac{y^{4}}{b^{2}}-1$ bounds a compact region, yet the Basic Problem is answered negatively by Corollary 4.1.1

Example 4.2. The curve defined by $\psi=y(y-x)(y+x)-x$ satisfies the necessary conditions from Theorem 4.1 One may show that the Basic Problem is affirmatively answered for all data $f \in \mathcal{P}_{2}$. For example, for $f=x^{2}$, the lowest degree solution is $u=x y\left(y^{2}-x^{2}\right)$. Note that $\operatorname{deg} u>\operatorname{deg} f$.

Corollary 4.1.1 compares with Proposition 2.2 of Hansen and Shapiro [7] for the Restricted Problem. The authors conjecture the following:

Conjecture 4.1. If $\psi$ is square-free and $\operatorname{deg} \psi>2$, then there is a negative answer to the Basic Problem. 


\section{ACKNOWLEDGEMENT}

This research was principally done while the first author visited the University of Waterloo in the summer of 1997. He gratefully acknowledges the financial support of D.S. during this period. The second author was supported by a grant from the Natural Sciences and Engineering Research Council of Canada.

The authors also wish to express their thanks to the anonymous referees for valuable suggestions and information regarding the literature.

\section{REFERENCES}

[1] S. Axler, P. Bourdon, and W. Ramey. Harmonic Function Theory. Springer-Verlag, New York, 1992. MR 93f:31001

[2] S. Axler and W. Ramey. Harmonic polynomials and Dirichlet-type problems. Proceedings of the Amer. Math. Soc., 123(12):3765-3773, 1995. MR 96b:31003

[3] P. Ebenfelt. Singularities encountered by the analytic continuation of solutions to Dirichlet's problem. Complex Variables Theory Appl., 20:75-91, 1992. MR 95f:30059

[4] P. Ebenfelt and H. S. Shapiro. The Mixed Cauchy Problem for Holomorphic Partial Differential Equations. J. D'Analyse Math., 65:237-295, 1995. MR 96f:35009

[5] L. Flatto and D. Newman and H. S. Shapiro. The Level Curves of Harmonic Functions. Transactions of the Amer. Math. Soc., 123:425-436, 1966. MR 33:5918

[6] L. Hansen and H. S. Shapiro. Graphs and Functional Equations. Ann. Acad. Sci. Fennicae, 18:125-146, 1993. MR 94b:30030

[7] L. Hansen and H. S. Shapiro. Functional Equations and Harmonic Extensions. Complex Variables, 24:121-129, 1994. MR 95f:30003

[8] H. S. Shapiro. An algebraic theorem of E. Fisher, and the holomorphic Goursat problem. Bull. London Math. Soc., 21:513-537, 1989. MR 90m:35008

[9] D. Khavinson and H. S. Shapiro. Dirichlet's problem when the data is an entire function. Bull. London Math. Soc., 24:456-468, 1992. MR 94d:35005

[10] M. H. Protter and H. F. Weinberger Maximum Principles in Differential Equations. SpringerVerlag, 1984. MR 86f:35034

[11] I. G. Shafarevich. Basic Algebraic Geometry 1, Second Edition. Springer-Verlag, New York, 1994. MR 95m:14001

Department of Mathematics and Computer Science, Grinnell College, Grinnell, IowA 50112-0806

E-mail address: chamberl@math.grin.edu

Department of Applied Mathematics, University of Waterloo, Waterloo, Ontario, CANADA N2L 3G1

E-mail address: dsiegel@math.uwaterloo.ca 